\title{
Impact of Thoracic Epidural Analgesia on Major Adverse Cardiac Events (Maces) Following Major Gastrointestinal Cancer Surgeries
}

\author{
Alaa Ali M. Elzohry ${ }^{1 *}$, Mohamad Farouk. Mohamad ${ }^{1}$, Ahmed Ali Obiedallah ${ }^{2}$ \\ Ahmed Fathy Abdel-Latif ${ }^{3}$, Ali Taha Abdel-Wahab ${ }^{4}$ \\ ${ }^{1}$ Department of Anesthesia, ICU and Pain Relief, South Egypt Cancer Institute, Assiut University, Egypt. \\ ${ }^{2}$ Department of Internal Medicine, Faculty of Medicine, Assiut University, Egypt. \\ ${ }^{3}$ Department of Anesthesia, ICU and Pain Relief, Faculty of Medicine, South Valley University, Egypt. \\ ${ }^{4}$ Department of Anesthesia and ICU, Faculty of Medicine, Minia University, Egypt. \\ alaa.zohiry@hotmail.com
}

*Corresponding Author: Alaa Ali M. Elzohry, MD., Department of Anesthesia, ICU and Pain Relief, South Egypt Cancer Institute, Assiut University, Arab Republic of Egypt.

Abstract

Background and Objectives: Major gastrointestinal cancer surgeries are associated with significant perioperative mortality and morbidity due to increased incidence of major perioperative cardiovascular event (MACEs). This study examined the effect of perioperative patient controlled epidural analgesia (PCEA) on reduction of MACEs in cardiac risky patients undergoing major gastrointestinal cancer surgery.

Methods: 60 patients (ASA II and III) of either sex were scheduled for elective Upper gastrointestinal cancer surgeries. Patients were allocated randomly into two groups (30 patients each) to receive, beside GA: continuous intra and post -operative intravenous infusion with fentanyl for 72 hours post-operatively (control group) or continuous intra and post -operative epidural infusion with bupivacaine $0.125 \%$ and fentanyl (TEA group) for 72 hours post-operatively

Intra-operative and post operative (HR and MAP) were recorded. Postoperative pain was assessed over $72 \mathrm{~h}$ using numerical rating scale (NRS). All patients were screened for occurrence of MACEs by ECG and echocardiography. And other postoperative complications and duration of ICU stay were recorded.

Results: There was a significant decrease in the incidence of MACEs with less pain scores in patients of TEA group in comparison to control group. Postoperative complications were comparable in both groups.

Conclusion: Perioperative PCEA in cardiac risky patients subjected to major gastrointestinal cancer surgery reduced significantly postoperative major adverse cardiac events with better pain control in comparison with perioperative PCIA analgesia.

Keywords: major perioperative cardiovascular event (MACEs), thoracic epidural analgesia, PCA, NRS.

\section{INTRODUCTION}

About 200 million surgeries were performed per year worldwide and the number is increasing. Of these patients, millions of major surgical procedures are performed each year and major gastrointestinal cancer surgery are typical representatives of such high-risk patients [1].
The high risk non-cardiac surgical population represents a major global healthcare challenge. And about 10 million patients suffer a major perioperative cardiovascular event (MACEs) within 30 days of perioperative period. [2] This is because elective major gastrointestinal urgery associated with severe postoperative morbidity (including cardiac ischemic 
Impact of Thoracic Epidural Analgesia on Major Adverse Cardiac Events (Maces) Following Major Gastrointestinal Cancer Surgeries

events) may not able to undergo subjective measures of cardiorespiratory reserve, such as metabolic equivalency tests (METs) [3].

Major adverse cardiac events (MACEs) as (non-fatal cardiac arrest, non-fatal myocardial infarction, heart failure, and clinically relevant arrhythmias), represent the most common cause of perioperative morbidity and mortality, with incidence rates ranging between $1 \%$ and $7 \%$. Despite decades of research into event prediction and prevention, the incidence of the events has remained largely unchanged [4].

Underlying cardiovascular disease significantly contributes to perioperative morbidity and mortality, and type of surgery, age of patients, cerebrovascular disease or diabetes mellitus increase overall prevalence of MACEs in non cardiac surgery being highest in patients older than $70 \mathrm{yr}$ undergoing major vascular surgery [5].

Moreover, anticancer chemotherapy is known to be cardiotoxic and itappears that chemotherapyincreases the postoperative cardiac risk after major cancer surgery. The most frequent cytotoxic agents used in treatment are cisplatin and 5-fluorouracil which have nephrotoxic and cardiotoxic effects, respectively. Additionally, combined use of chemotherapy and radiation is associated with higher treatment-related toxicities than use of radiation alone [6].

Cardiac Risk Index (RCRI) is one of the most widely used cardiac event risk stratification system and a lot of literature addressing the impact of intraoperative anesthetic hemodynamic management on perioperative cardiac events. [7-8].

Epidural analgesia is effectively applied in these surgeries to improve perioperative pain; epidural analgesia is coupled with improved analgesia, earlier extubation time, better haemodynamics, less respiratory complications, and superior left ventricular function [9-11].

The aim of this study was to evaluate the effect of perioperative patient controlled epidural analgesia (PCEA) on reduction of (MACEs) in comparison to patient controlled intravenous analgesia (PCA) in risky cardiac patients who were undergone major upper gastrointestinal cancer surgeries.

\section{Patients And Methods}

This prospective randomized study was approved by the local ethics committee of the South Egypt Cancer Institute, Assiut University, Egypt. In the duration from October 2017 till October 2018, after written informed consent, ASA (II-III) 66 ischemic cardiac adult patients were randomly allocated for elective major gastrointestinal cancer but 60 patients of them were finally analyzed.

Exclusioncriteriawere the following: contraindications to epidural catheter insertion (coagulopathy, recent -less than 1 week-treatment with thrombolytic or potent anti platelet drugs as clopidogrel, and local infection), allergy to local anesthetic medications or opioids. Patient whose ability to use PCEA pump or who cannot be taught how to evaluate their own pain intensity were also excluded from the study.

All patients were evaluated preoperatively by a cardiologist and anesthesiologist according to the revised cardiac risk index -Lee RCRI index -. That was calculated as one point was assigned to each of the following factors: a history of CAD, a history of cerebrovascular disease, history of heart failure, insulin-dependent diabetes mellitus, impaired renal function, and high-risk type of surgery. ECG and echocardiography were recorded and analyzed.

Preoperative data were taken within two days before surgery as; demographic data, medical, surgical history, physical examination and routine laboratory investigations. One day before surgery, all patients were taught how to evaluate their own pain intensity using the Numerical Rating Scale (NRS), scored from $0-10$ (where $0=$ no pain and $10=$ worst pain imaginable).and how to use the PCA device (Abbott Pain Management Provider. S. No: 96450292. Abbott Laboratory, North Chicago. IL: 60064, USA) ®.

The Patients were randomly assigned into two groups (30 patients each) by using opaque sealed envelopes containing computer generated randomization schedule, the opaque sealed envelopes are sequentially numbered that were open before application of anesthetic plan.

Patients of both groups were premedicated with midazolam $0.05 \mathrm{mg} / \mathrm{kg}$ and ranitidine $50 \mathrm{mg}$. After shifting the patient to the induction room , ECG, pulse oximeter, non-invasive blood pressure and invasive 
Impact of Thoracic Epidural Analgesia on Major Adverse Cardiac Events (Maces) Following Major Gastrointestinal Cancer Surgeries

blood pressure monitors were attached. Peripheral Venous line and an infusion of lactated ringers' solution was started.

\section{Control group (No. $=30)$}

-Surgery was performed under standard general anesthesia.

-Postoperative analgesia was provided through patient Intravenous -controlled analgesia (PICA) for 72 hours postoperatively.

TEA group (No. $=30$ )

-Surgery was done under standard general anesthesia. And additionally Thoracic Epidural catheter was inserted prior induction of GA.

Postoperative analgesia will provided through PatientControlled Epidural Analgesia (PCEA) using TEA for 72 hours postoperatively.

\section{Standard General Anesthesia}

After pre-oxygenation for 3 minutes, intravenous anesthesia (propofol $1.5 \mathrm{mg} / \mathrm{kg}$ ) induced with fentanyl 1-2 $\mu \mathrm{g} / \mathrm{kg}$ administered over min. Tracheal intubation will be performed after adequate neuromuscular blockade with cisatracurium 0.5 $\mathrm{mg} / \mathrm{kg}$. Anesthesia was maintained by isoflurane 1-1.5 MAC, cisatracurium $0.03 \mathrm{mg} / \mathrm{kg}$ given when indicated. Patients were mechanically ventilated to maintain ETCO2 between 35-40 mmHg. The inspired oxygen fraction (FIO2) was 0.5 using oxygen-and-air mixtures. At the end of surgery neuromuscular block was antagonized in all patients with neostigmine 0.05 $\mathrm{mg} / \mathrm{kg}$ and atropine $0.02 \mathrm{mg} / \mathrm{kg}$ and trachea was extubated in the operating room. Tracheal extubation were performed when patients meet the following criteria: hemodynamic stability, adequate muscle strength, full consciousness, and adequate ventilation breathing rate: 10 to 30 breaths/min, $\mathrm{PaO} 2 / \mathrm{IFO} 2$ $\geq 80 / 0.4, \mathrm{PaCO} 2,30$ to $45 \mathrm{mmHg}$ ).

Intra operative analgesia in control group: - by continuous intravenous fentanyl infusion $2 \mu \mathrm{g} / \mathrm{kg} /$ hr intra operatively to maintain heart rate (HR) and blood pressure within $20 \%$ of the basal value. Rescue analgesia of $0.5 \mu \mathrm{g} / \mathrm{kg}$ was given. Fentanyl infusion was continued until shifting the patient to ICU.

IntraoperativeanalgesiainTEAgroup:byepiduralbolus dose of $0.1 \mathrm{ml} / \mathrm{kg}$ of a mixture $(0.125 \%$ bupivacaine /
Fentanyl $10 \mu \mathrm{g} / \mathrm{ml}$ ) After a negative response to test dose-was administered, epidural were considered to be adequately working if there is decreased pin prick sensation at the expected dermatomal level, decreased blood pressure from its basal level and absence of stress response to surgical incision. Then, the bolus dose was followed by continuous infusion of $0.1 \mathrm{ml} /$ $\mathrm{kg}$ of the same mixture.

\section{THORACIC EPIDURAL CATHETER}

Under strict aseptic precautions thoracic epidural was performed using a 16 gauge Tuhy epidural needle by a paramedian approach. T8-T9 interspace was chosen for the injection (with air) after skin wheal of lidocaine local anesthetic 2\%. The catheter was introduced approximately $4 \mathrm{~cm}$ into the epidural space. The epidural space was identified by the loss of resistance technique. A $3 \mathrm{ml}$ test dose of $2 \%$ Lidocaine with 1 : 200,000 Adrenaline was given after the placement of the epidural catheter.

Post operative analgesia in control group; using PCA Fentanyl $10 \mu \mathrm{g} / \mathrm{ml}$ solutions through PCA device that programmed to give a bolus dose $2 \mathrm{ml} /$ dose with a minimal lockout interval of $10 \mathrm{~min}$ with no background infusion. Post operative analgesia in TEA group using PCEA; background epidural infusion of $0.1 \mathrm{ml} / \mathrm{kg} / \mathrm{h}$ of the mixture of $(1.25 \mathrm{mg} / \mathrm{ml}$ bupivacaine plus $10 \mu \mathrm{g}$ /ml Fentanyl) and $3 \mathrm{ml}$ as top up dose of this mixture with lockout interval of $20 \mathrm{~min}$. The analgesic regimen in both groups was adjusted to achieve a NRS $<3$.

Data collected Intra operatively include (MAP, HR, colloid, and blood transfusion,) and duration of anesthesia and surgery.

Post operative all patients were admitted to surgical ICU and were followed-up by an investigator who was blinded to the study and findings on ECG. And beside the routine follow up, the following were recorded:

-Daily 12-lead ECG for 3days,

-Vital signs (HR, MAP and CVP) were recorded every one hour in ICU.

-Echocardiography to evaluate cardiac function.

-NRS- every 4 hours for 3 days-for pain measurement. And total fentanyl consumption was calculated.

-Any concomitant events like nausea; vomiting, pruritus or respiratory depression (decrease oxygen 
Impact of Thoracic Epidural Analgesia on Major Adverse Cardiac Events (Maces) Following Major Gastrointestinal Cancer Surgeries

saturation $\geq 90 \%$ ) were recorded postoperatively.

-Duration of hospital and ICU stay and patients outcome (living or dead).

Study end-points:-Occurrence one or more of MACE (major advanced cardiac events) such as myocardial ischemia, myocardial infarction [MI], pulmonary edema, Non-fatal cardiac arrest, primary cardiac arrest, and malignant arrhythmia throughout 72 hours after surgery.

Non-fatal cardiac arrest: An absence of cardiac rhythm or presence of chaotic rhythm requiring any component of basic or advanced cardiac life support.

Acute myocardial infarction and ischaemia were replaced by terminology of myocardial injury which is more sensitive to evidence of myocardial cell necrosis. And also infarction and ischaemia are difficult to define in this setting because patients often do not have the classical symptoms or ECG changes of myocardial infarction.

We defined myocardial injury as (new ECG findings suggestive of ischemia, new ST segment changes, new pathologic $\mathrm{Q}$ wave or new $\mathrm{T}$ wave inversion) or New echocardiographic findings suggestive of ischemia (new regional wall motion abnormalities).

Congestive heart failure was defined as new inhospital signs or symptoms of dyspnoea or fatigue, orthopnoea, paroxysmal nocturnal dyspnoea, increased jugular venous pressure, pulmonary rales on physical examination, cardiomegaly, or pulmonary vascular engorgement. The decision cut-point for the diagnosis of heart failure is identical to that of $100 \mathrm{pg} / \mathrm{ml}$.

Malignant arrhythmia was defined as ECG evidence of atrial flutter, atrial fibrillation, or second- or thirddegree atrioventricular conduction Block.

All ECGs and Echocardiography were analyzed by a consultant cardiologist who was responsible for the patients' management.

\section{STATISTICAL ANALYSIS}

The required sample size was calculated using Epi Info software version 7 (CDC, 2012) ®. Using post hoc power analysis with accuracy mode calculations with incidence of MACEs as the primary objective and therefore, it was estimated that minimum sample size of 29 patients in each study group would a chive a power of $80 \%$ to detect an effect size of 0.8 in the outcome measures of interest, assuming a type I error of 0.05

All analyses were performed with the SPSS 21.0 (R) software. Categorical variables were described by number and percent (N, \%), where continuous variables described by mean and standard deviation (Mean, SD). And Mann-Whitney test were used to compare between two groups while Chi square test was used for qualitative data. Where compare between continuous variables by t-test. $\mathrm{P}$ was considered significant if 60.05 at confidence interval 95\%.

\section{RESULTS}

66 ischemic cardiac adult patients were randomly allocated for elective major abdominal cancer but 60 patients of them were finally analyzed (Figure 1) with no statistical differences in demographic data (table1) nor pre-operative morbid conditions (table 2) and echo findings (table $3 \&$ Figure 2).

In our study, the main finding was decreased of perioperative MACEs in patients of the TEA group in comparison to patients of control group (Figure 4).

In patients of the TEA group the quality of postoperative analgesia was better especially during first 24 hour postoperative period in comparison to control group (table 6), with comparable other postoperative side effects as (nausea, vomiting and pruritus) between two groups during 72 hours (Figure 5).

HR and MAP also was optimized significantly both intra and post operatively in TEA group instead of being elevated (not markedly but significantly) in control group (table 4, 5). Significant increase in ICU and hospital stay, in patients of control group who also showed increased consumption fentanyl in post operative period in comparison to patients of TEA group (table 7).

There were no cases of serious epidural catheterrelated complications, such as respiratory depression, epidural hematoma or abscess, local inflammation, or permanent neurologic damage in our study. 
Impact of Thoracic Epidural Analgesia on Major Adverse Cardiac Events (Maces) Following Major Gastrointestinal Cancer Surgeries

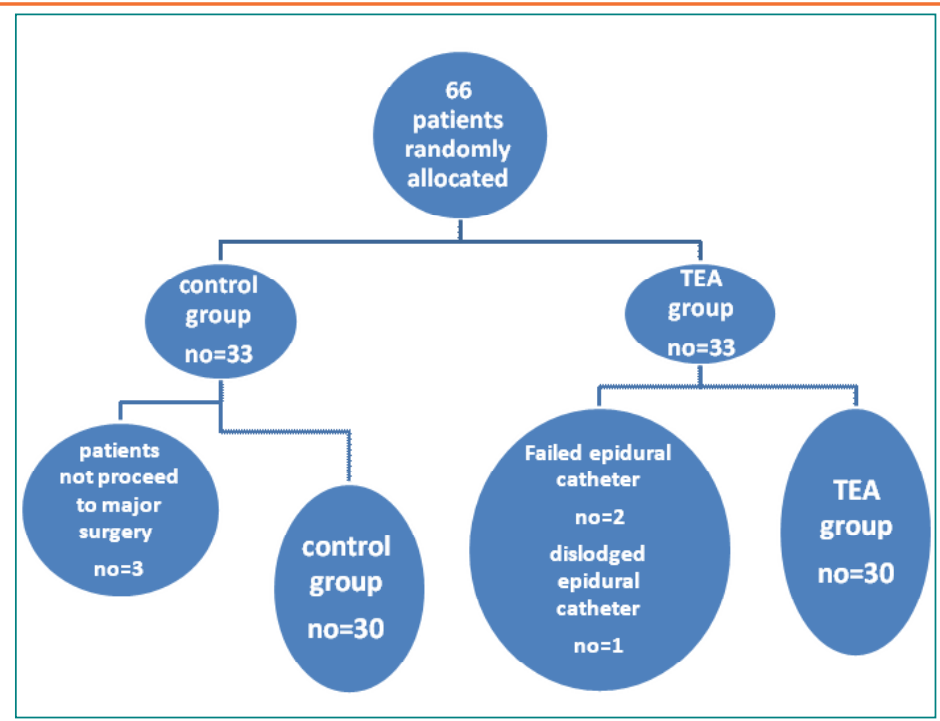

Figure 1. Flow diagram of patients

Table 1. Demographic data and patient's characteristics

\begin{tabular}{|l|l|l|l|}
\hline \multicolumn{1}{|c|}{} & \multicolumn{1}{|c|}{ Control group (n=30) } & \multicolumn{1}{c|}{ TEA group (n=30) } & \multicolumn{1}{c|}{ P. value } \\
\hline Age: mean \pm SD & $63.73 \pm 5.61(55-74)$ & $61.73 \pm 6.07(5574)$ & 0.191 \\
\hline Gender, M/F & $18 / 20$ & $12 / 10$ & 0.592 \\
\hline BMI, kg/m 2: mean \pm SD & $22.1 \pm 3.3$ & $23.9 \pm 1.5$ & 0.066 \\
\hline ASA (n and \%) & $14(46.7)$ & $12(40.0)$ & 0.501 \\
\hline II & $16(53.3)$ & $9(60.0)$ & 0.196 \\
\hline III & $5.64 \pm 0.7(4.4-7)$ & $5.41 \pm 0.68(4.3-7)$ & \\
\hline $\begin{array}{l}\text { Operative duration (hours) } \\
\text { mean } \pm \text { SD }\end{array}$ & \multicolumn{2}{l|}{} \\
\hline Type of Surgery: & $17(56.7 \%)$ & $15(50.0 \%)$ & 0.795 \\
\hline - Whipple's surgery & $7(23.3 \%)$ & $8(26.7 \%)$ & 0.998 \\
\hline - Gastrectomy & $6(20.0 \%)$ & $7(23.3 \%)$ & 0.976 \\
\hline - Oesophagectomy & \multicolumn{2}{|l|}{} \\
\hline
\end{tabular}

Data were expressed as mean $\pm \mathrm{SD}$., TEA =thoracic significant. Between two groups no significant epidural group. P. value $<0.05$ considered statistically difference found regarding patient's characteristics.

Table 2. Patient's pre-operative morbid conditions

\begin{tabular}{|l|l|l|l|}
\hline & Control group (n=30) & TEA group (n=30) & P. value \\
\hline ECG finding: & $10(33.3 \%)$ & $11(36.7 \%)$ & 0.782 \\
\hline Atrial fibrillation & $4(13.3 \%)$ & $4(13.3 \%)$ & 1.000 \\
\hline Pre mature atrial ectopics & $2(6.7 \%)$ & 0.394 \\
\hline Bundle branch block & $4(13.3 \%)$ & $11(36.7 \%)$ & 0.782 \\
\hline Ventricular Hypertrophy & $10(33.3 \%)$ & $14(46.7 \%)$ & 1.000 \\
\hline Site of Ischemic Heart Disease (IHD) & $14(46.7 \%)$ & $0(0 \%)$ & 0.374 \\
\hline Inferior IHD & $2(6.7 \%)$ & \\
\hline Anterior IHD &
\end{tabular}

Archives of Anesthesiology V2 . I1 . 2019 
Impact of Thoracic Epidural Analgesia on Major Adverse Cardiac Events (Maces) Following Major Gastrointestinal Cancer Surgeries

\begin{tabular}{|c|c|c|c|}
\hline Lateral IHD & $8(26.7 \%)$ & $4(13.3 \%)$ & 0.194 \\
\hline Mixed IHD & $6(20 \%)$ & $12(40 \%)$ & 0.091 \\
\hline \multicolumn{4}{|l|}{ Risky diseases : } \\
\hline Hypertension & $15(50 \%)$ & $16(53.3 \%)$ & 0.798 \\
\hline Diabetes Mellitus & $16(53.3 \%)$ & $15(50 \%)$ & 0.798 \\
\hline Renal Failure & $4(13.3 \%)$ & $5(16.7 \%)$ & 0.712 \\
\hline COPD & $2(6.7 \%)$ & $3(10 \%)$ & 0.644 \\
\hline Hyper lipedemia & $14(46.7 \%)$ & $12(40 \%)$ & 0.601 \\
\hline RCSI, mean \pm SD (range) & $3.07 \pm 0.78(2-4)$ & $3.07 \pm 0.58(2-4)$ & 0.782 \\
\hline
\end{tabular}

Data were expressed as mean $\pm \mathrm{SD}$., TEA $=$ thoracic significant. Between the two groups no significant epidural group. P. value $<0.05$ considered statistically difference found regarding patient's characteristics.

Table 3. patient's pre-operative Echo findings

\begin{tabular}{|c|c|c|c|}
\hline & Control group $(n=30)$ & TEA group $(n=30)$ & P. value \\
\hline \multicolumn{4}{|l|}{ Diastolic dysfunction } \\
\hline Grade 1(G1) & $22(73.3 \%)$ & $24(80 \%)$ & 0.834 \\
\hline Grade 2 (G2) & $8(26.7 \%)$ & $6(20 \%)$ & 0.705 \\
\hline \multicolumn{4}{|l|}{ VALVE LESION } \\
\hline No valve lesion & $7(23.3 \%)$ & $7(23.3 \%)$ & 1.000 \\
\hline Mild valve lesion & $10(33.3 \%)$ & $9(30 \%)$ & 0.783 \\
\hline Moderate lesion & $12(40 \%)$ & $13(43.3 \%)$ & 0.795 \\
\hline Sever valve regurge & $1(3.3 \%)$ & $1(3.3 \%)$ & 1.000 \\
\hline \multicolumn{4}{|l|}{ Other ECHO findings } \\
\hline SWMA & $8(26.7 \%)$ & $9(30 \%)$ & 0.777 \\
\hline Dilated atrium or ventricle & $14(46.7 \%)$ & $13(43.3 \%)$ & 0.791 \\
\hline Pulmonary hypertension & $9(30 \%)$ & $11(36.7 \%)$ & 0.582 \\
\hline Hyper trophic ventricle & $10(33.3 \%)$ & $8(26.7 \%)$ & 0.577 \\
\hline EF, mean \pm SD (range) & $49.8 \pm 5.99(41-60)$ & $48.4 \pm 5.52(39-59)$ & 0.789 \\
\hline
\end{tabular}

Data were expressed as mean \pm SD. SWMA epidural group. EF=ejection fraction, between two =segmental wall motion abnormality, TEA =thoracic groups no significant difference was found

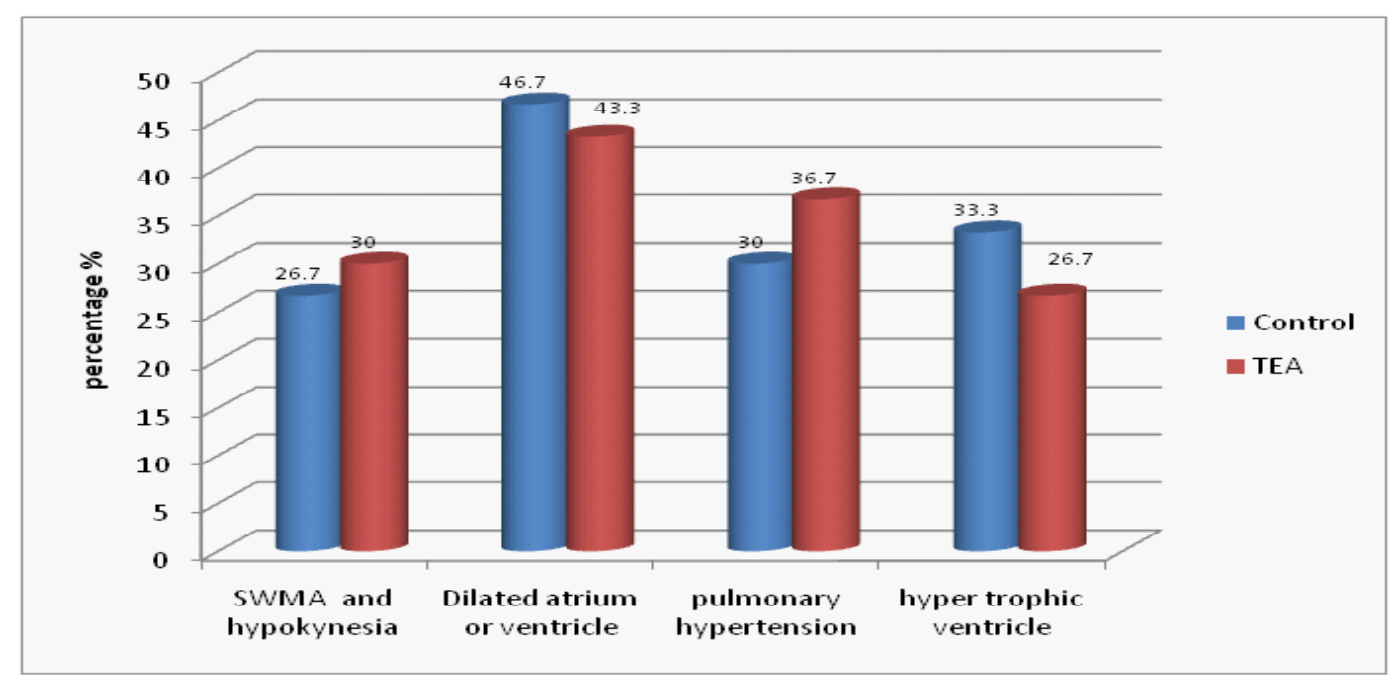

Figure 2. pre-operative Other ECHO findings 
Impact of Thoracic Epidural Analgesia on Major Adverse Cardiac Events (Maces) Following Major Gastrointestinal Cancer Surgeries

SWMA= segmental wall motion abnormality, TEA significant difference found.

$=$ thoracic epidural group. between two groups no

Table 4. Intra-operative haemodynamics

\begin{tabular}{|l|l|l|l|}
\hline \multicolumn{1}{|c|}{ Intraoperative MAP (mmhg) } & \multicolumn{1}{|c|}{ Control group (n=30) } & \multicolumn{1}{|c|}{ TEA group (n=30) } & \multicolumn{1}{|c|}{ P. value } \\
\hline Baseline reading & $75.9 \pm 10.6(58-94)$ & $80 \pm 9.3(68-95)$ & 0.117 \\
\hline 1h & $82.47 \pm 10.04(62-100)$ & $73.53 \pm 10.02(56-90)$ & $0.002^{*}$ \\
\hline 2h & $79.67 \pm 12.12(62-98)$ & $65.07 \pm 7(52-77)$ & $0.001^{*}$ \\
\hline 3h & $75.73 \pm 11.79(60-100)$ & $69.67 \pm 7.32(60-82)$ & 0.120 \\
\hline 4h & $77.2 \pm 13.43(62-108)$ & $73.2 \pm 8.35(59-86)$ & 0.218 \\
\hline 5h & $73.13 \pm 8.86(62-98)$ & $72.93 \pm 4.95(65-82)$ & 0.914 \\
\hline 6h & $75.73 \pm 11.79(60-100)$ & $69.67 \pm 7.32(60-82)$ & 0.120 \\
\hline Mean & $\mathbf{7 7 . 8 5} \pm \mathbf{9 . 0 2}(\mathbf{6 3 . 8}-\mathbf{9 8})$ & $\mathbf{7 0 . 0 7} \pm \mathbf{5 . 6 5}(\mathbf{6 2 - 8 1 . 8 )}$ & $\mathbf{0 . 0 1 8}$ \\
\hline Intraoperative HR (bpm) & $72.8 \pm 11.0(56-94)$ & $77.9 \pm 14.0(57-97)$ & 0.126 \\
\hline Baseline reading & $85.13 \pm 10.37(65-98)$ & $75.4 \pm 7.16(65-89)$ & $0.001^{*}$ \\
\hline 1h & $82.93 \pm 18.02(56-120)$ & $72.47 \pm 14.43(57-110)$ & $0.016^{*}$ \\
\hline 2h & $82.27 \pm 13.96(58-110)$ & $72.67 \pm 11.81(56-108)$ & $0.012^{*}$ \\
\hline 3h & $80.07 \pm 14.14(59-108)$ & $75.33 \pm 11.57(50-98)$ & 0.161 \\
\hline 4h & $72.93 \pm 12.34(55-100)$ & $72.73 \pm 13.05(55-100)$ & 0.952 \\
\hline 5h & $80.07 \pm 14.14(59-108)$ & $76.33 \pm 11.57(50-98)$ & 0.168 \\
\hline 6h & $\mathbf{8 0 . 6 7 + 8 . 9 2 ( 6 8 . 2 - 9 6 . 2 )}$ & $\mathbf{7 3 . 7 2 + 8 . 4 7 ( 6 6 . 2 + 1 0 1 . 0 )}$ & $\mathbf{0 . 0 1 6 *}$ \\
\hline Mean &
\end{tabular}

Data are expressed as mean \pm SD. At base line reading groups there was significant difference in early post and $1,2,3,4,5$ and 6 hours MAP= mean arterial pressure (mmhg), HR=heart rate (beat per minutes).h=hour operative periods.being optemized in TEA group and elevated in control. interval TEA =thoracic epidural group.between two

Table 5. Post-operative haemodynamic variables (mean of readings/day)

\begin{tabular}{|c|c|c|c|c|c|}
\hline & \multicolumn{2}{|c|}{ Control group $(n=30)$} & \multicolumn{2}{|c|}{ TEA group $(n=30)$} & \multirow[t]{2}{*}{ P. value } \\
\hline & Range & Mean \pm SD & Range & Mean \pm SD & \\
\hline \multicolumn{6}{|c|}{ Post-operative HR (bpm) } \\
\hline Day1 & $64.8-148.4$ & $92.91 \pm 19.07$ & $55-128$ & $84.59 \pm 21.78$ & $0.003^{*}$ \\
\hline Day2 & $67.4-121.4$ & $88.53 \pm 15.1$ & $63.6-112$ & $80.6 \pm 15.14$ & $0.047^{*}$ \\
\hline Day3 & $68.8-83.6$ & $85.11 \pm 7.14$ & $61.4-83.8$ & $77.41 \pm 4.63$ & 0.247 \\
\hline \multicolumn{6}{|c|}{ Post-operative MAP (mmhg) } \\
\hline Day1 & $68.8-83.6$ & $77.21 \pm 4.63$ & $61.4-83.8$ & $73.69 \pm 7.14$ & $0.031^{*}$ \\
\hline Day2 & $64.8-87.2$ & $76.15 \pm 7.94$ & $62.6-89.2$ & $73.75 \pm 7.96$ & 0.247 \\
\hline Day3 & $58.4-88.4$ & $72.37 \pm 9.72$ & $59-87$ & $71.2 \pm 8.3$ & 0.617 \\
\hline \multicolumn{6}{|c|}{ Post-operative CVP (cm H2O) } \\
\hline Day1 & $5.2-19$ & $8.89 \pm 2.72$ & $5.2-16$ & $10.01 \pm 3.7$ & 0.187 \\
\hline Day2 & $4.2-19.8$ & $8.47 \pm 1.86$ & $6.4-13.8$ & $9.76 \pm 3.89$ & 0.106 \\
\hline Day3 & $7-15.6$ & $8.13 \pm 1.59$ & $6.6-13.2$ & $8.97 \pm 2.13$ & 0.093 \\
\hline
\end{tabular}

Archives of Anesthesiology V2 . I1 . 2019 
Impact of Thoracic Epidural Analgesia on Major Adverse Cardiac Events (Maces) Following Major Gastrointestinal Cancer Surgeries

Data were expressed as mean \pm SD. HR=heart rate (beat per minutes). TEA =thoracic epidural group. $\mathrm{MAP}=$ mean arterial pressure $(\mathrm{mmhg}), \mathrm{CVP}=$ central venous pressure ( $\mathrm{cmH} 2 \mathrm{o})$. between two groups there Table 6. Post-operative NRS (3 days readings) was ONLY significant difference in early post operative day regarding patient's H.R and MAP but not CVP. P. value $<0.05$ considered statistically significant

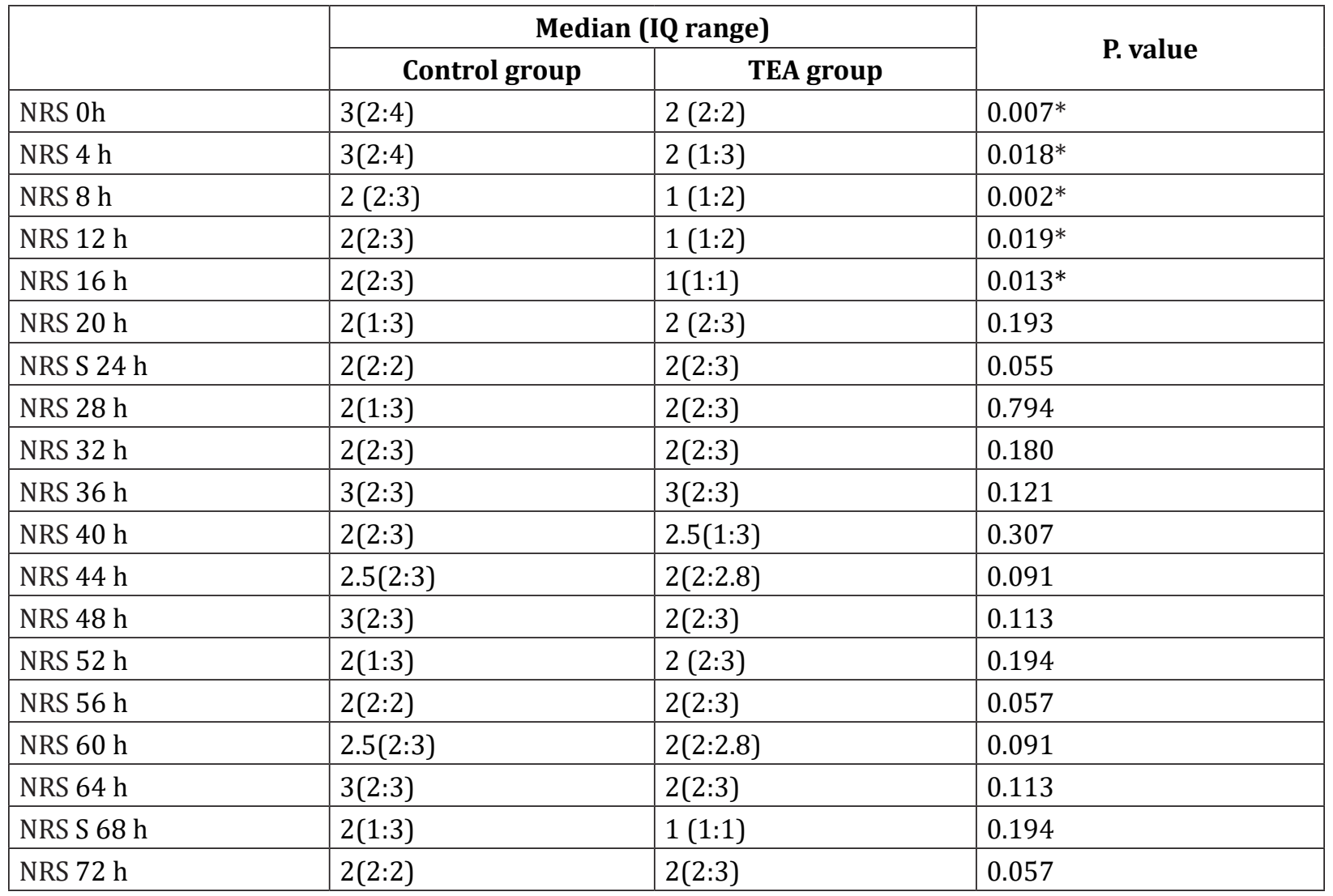

Data were expressed as Median and (IQ range). between two groups there was significant difference VAS=visual analogue scale TEA =thoracic epidural in early post operative hours P. value $<0.05$ considered group.0h=immediately after recovery, $\mathrm{h}=$ hour. statistically significant.

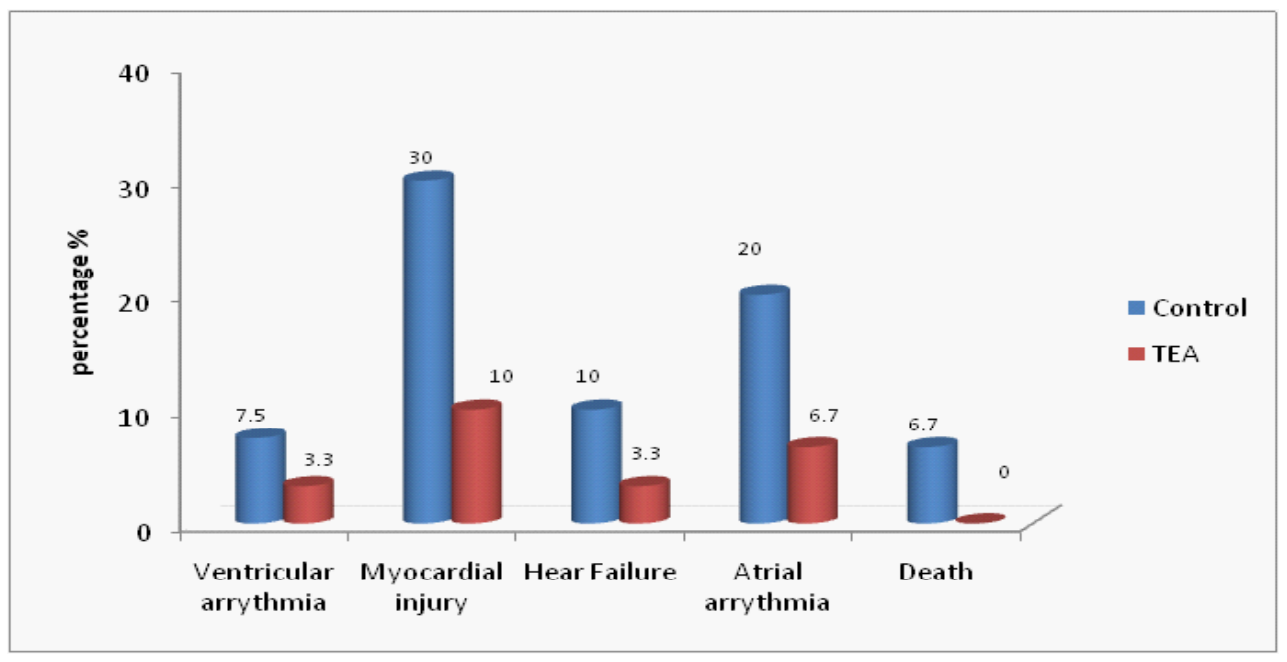

Figure 3. Post operative MACE 
Impact of Thoracic Epidural Analgesia on Major Adverse Cardiac Events (Maces) Following Major Gastrointestinal Cancer Surgeries

Data were expressed in number and percentage. TEA =thoracic epidural group ,MACE = major advanced cardiac events, $\mathrm{HF}=$ heart failure, $\mathrm{EF}=$ ejection fraction. in postoperative period in TEA group there was significant decreased of myocardial injury and postoperative arrhythmia (atrial and ventricular). compared to control group. Moreover, there were trends towards decreased other complications as Congestive heart failure but did not reach statistical significance

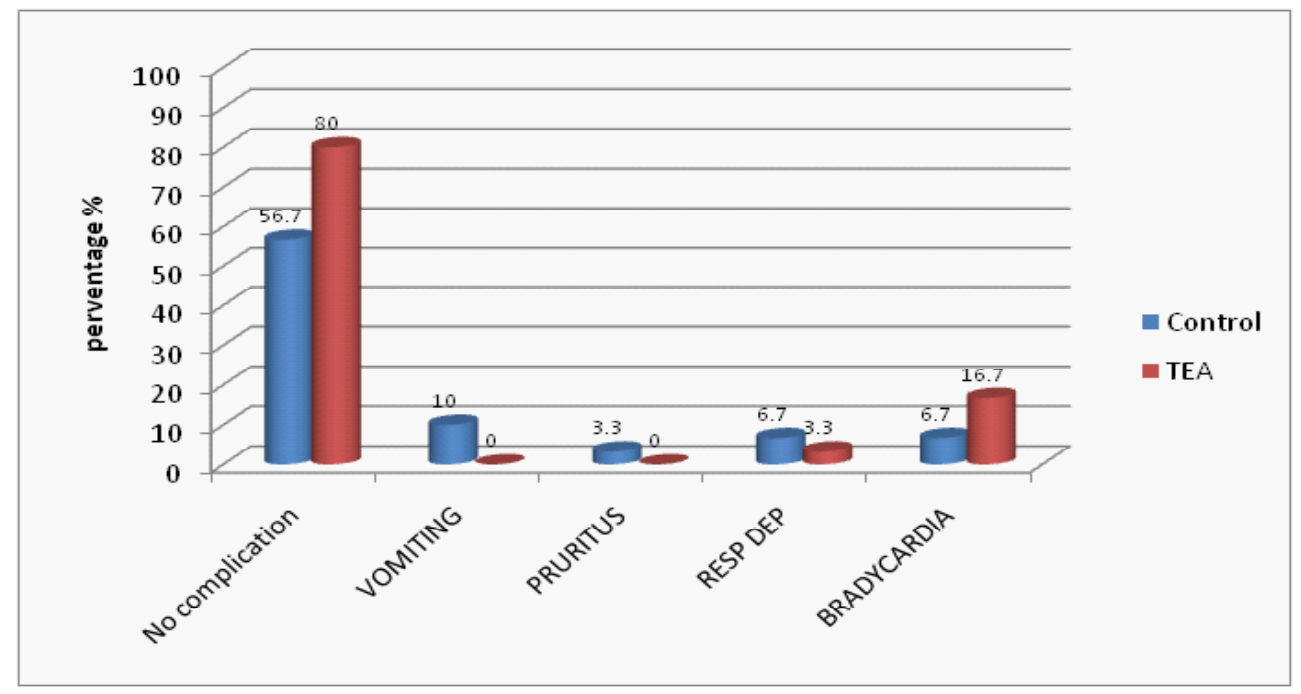

Figure 4. Post-operative complications from the studied drugs

TEA =thoracic epidural analgesia group, RESP difference between two groups. $\mathrm{DEP}=$ respiratory depression There was no significant

Table 7. ICU, Hospital stay and Fentanyl consumption

\begin{tabular}{|l|l|l|l|l|l|}
\hline & \multicolumn{2}{|c|}{ Control group $(\mathbf{n = 3 0})$} & \multicolumn{2}{c|}{ TEA group $(\mathbf{n = 3 0})$} & \multirow{2}{*}{ P. value } \\
\cline { 2 - 5 } & \multicolumn{1}{|c|}{ Range } & \multicolumn{1}{c|}{ Mean \pm SD } & \multicolumn{1}{c|}{ Range } & \multicolumn{1}{c|}{ Mean \pm SD } & \\
\hline ICU stay(day) & $3-11$ & $7.47 \pm 2.16$ & $3-8$ & $5.6 \pm 1.57$ & $0.00^{* *}$ \\
\hline Hospital stay(day) & $3-31$ & $22.13 \pm 7.62$ & $10-25$ & $18.13 \pm 4.12$ & $0.014^{*}$ \\
\hline $\begin{array}{l}\text { Fentanyl (mic/72h) } \\
\text { consumption }\end{array}$ & $1200-2000$ & $1646.67 \pm 234.5$ & $600-1000$ & $753.33 \pm 122.43$ & $0.000^{* *}$ \\
\hline
\end{tabular}

Data were expressed as mean $\pm \mathrm{SD}$, TEA =thoracic epidural analgesia group, ICU=intensive care unit P. value $<0.05$ considered statistically significant. There was significant difference between two groups. Patients of TEA group stay less period in ICU and hospital and consumed less fentanyl.

\section{DISCUSSION}

In patients of the TEA group the quality of postoperative analgesia was better especially during 72 hour postoperative period in comparison to control group. Our choice of 72 hours as period for PCA (either I.V. or epidural) because the large proportion of clinically unrecognized acute myocardial infarction (AMI) is related to the fact that most AMI occur during the early postoperative period [12].
In our study, the main finding was decreased of MACEs in patients of the TEA group in comparison to patients of control group.

Generally, major non-cardiac surgery is associated with Major advanced cardiac events which represent the most common cause of serious perioperative morbidity and mortality. One out of 10 patients who developed a MACE died during the hospital stay [13]. Most MACE-related death after major surgery arises from myocardial infarction and arrhythmias. [14].

MI is associated with poor prognosis. However, asymptomatic perioperative $\mathrm{MI}$ is as strongly associated as symptomatic MI with 30 day mortality. [15]. Other Authors observed low incidence of serious postoperative cardiac events in Major non-cardiac surgery as Mangano (3\%) and Lee (2\%) [16-17]. 
We can explain the high prevalence of cardiac complicationsinthemajorsurgeries-liketheseselected for the study-is due to the fact that major surgery triggers an organism's response to stress, which is characterized by profound endocrine, metabolic, and hemodynamic alterations. The activation of the sympathetic system results in tachycardia and increases myocardial oxygen consumption. [18].

Also major surgeries especially that carried out in upper abdomen are procedures with intense pain that, if not properly treated, may cause deep physiological and hormonal alterations in the body [19]. More over severe abdominal pain causes shallow breathing, atelectasis, retention of secretions and lack of cooperation in physiotherapy followed by hypoxia and cardiac complication. This increases the incidence of post-operative morbidity and leads to delayed recovery. [20].

Cardiac sympathetic over activity can damage myocardial cells by causing a release of large amount of catecholamines and cytokines. Therefore, locally blocking cardiac sympathetic fibers could considerably protect the heart and reduce the secondary effects. [21]. Both TEA and intravenous analgesia were found to reduce pulmonary and cardiac complications, and improve tissue oxygenation and tissue reperfusion. [22].

TEA was heavily studied and the utility of peri-operative epidural analgesia in reducing postoperative cardiac morbidity has created much controversy since Yeager et al. [23]. Then many studies (agree with us) have shown that TEA together with general anaesthesia provided better myocardial protection than general anaesthesia alone [24-25] and many meta-analyses suggest that TEA may decrease cardiac morbidity and mortality after cardiac and major non-cardiac surgery. [26-27]. and in experimental myocardial ischaemia, TEA reduced infarct size.

Other study that confirm our results proved that cardiac morbidity was shown to be lower among patients undergoing major vascular surgery after the administration of general anesthesia combined with postoperative epidural analgesia compared to the administration of general anesthesia alone and postoperative systemic opioid analgesia [28].

TEA was found to decrease adverse perioperative cardiac events by its overall reduction of sympathetic tone and block of the cardiac accelerator fibers could reduce the risk of dysrhythmias and injury as observed during cardiac surgery and cardiopulmonary bypass. [29]. Better pain relief with concomitant reduction in the postoperative stress response and systemic sympathetic activity may contribute to this effect [30].

More over Blomberg et al found that large coronary epicardial arteries and coronary arterioles are densely innervated by sympathetic adrenergic nerve fibers. Coronary blood flow after TEA was investigated in patients with ischemic heart disease. Endocardial to epicardial blood flow was improved, so that regional distribution of perfusion is optimized. [31]

Also Schmidt et al approved that TEA improved diastolic function in patients with coronary artery disease undergoing operative revascularization. Diastolic dysfunction has been reported to be an early sign of cardiac ischaemia. [32].

On contrary of our results; clinical data on myocardial ischaemia and mortality are inconclusive as in a randomized trial that showed TEA did not reduce the 30 day complication rate and do not confirm that reduced morbidity such as respiratory or cardiac complication after cardiac surgery. [33]

Also against us a study conducted by Mohamed et al, in our institute they studied 60 ischemic patients underwent elective major abdominal cancer surgery assigned into 2 groups; 30 patients each to receive general anesthesia (G1) or combined general and epidural anesthesia (G2).they concluded that The LEA combined with general anesthesia in high risk patients with ischemic heart disease undergoing major abdominal cancer surgery provided better pain relief, and Ischemic cardiac events were similar in both groups. [34]

Regardinghaemodynamic; Kessler etal. who compared the heart rate in patients between those who received general anaesthesia together with TEA(Group1) and those who received only general anaesthesia (Group2) during coronary artery bypass surgery performed on a beating heart and reported that the heart rate in the group 1 was lower than preoperative values ,during sternotomy and anastomosis compared to group 2. [35]

But both Berendes et al. and Fillinger et al. [36-37]. were against us as they did not observe a difference in hemodynamic findings between the control group and 
Impact of Thoracic Epidural Analgesia on Major Adverse Cardiac Events (Maces) Following Major Gastrointestinal Cancer Surgeries

the TEA treatment group who studied TEA in patients undergoing coronary artery bypass grafting.

Scott et al. presented the first randomized evaluation of the impact of perioperative TEA on outcome in a large series of 400 patients with normal ventricular function undergoing $C A B G$ in whom the epidural catheters were placed immediately before surgery. There was a reduction in the incidence of supra ventricular arrhythmias (SVA). [38]

According to Moltner, Dysrhythmias are common complications in immediate postoperative period even more common after upper abdominal and thoracic surgeries [39]. As conducted by Giroban et al who concluded that dysrhythmias in the postoperative period was 20\% of 185 patients undergoing thoracobdominal surgeries. [40]

We can explain occurrence of arrhythmias by many factors as pre-existing cardiac pathology, intraoperative events, and arrhythmia triggers. Autonomic imbalance after operation has been implicated as a possible trigger, and is thought to be characterized by increased sympathetic tone and lower vagal tone.. Also Dysrhythmias may be associated with improper treatment of pain, and have the excess of circulating catecholamines as the most important physiopathological mechanism, producing an imbalance between oxygen offer and consumption by the myocardium. [41-42]

In our study, no patient developed significant hypotension. Similar findings were noted in studies done by Elzohry et al .In the study conducted by five episodes of postoperative hypotension occurred in the PCEA group versus none in the PCA group. The patients were treated by simple fluid loading. [43].

The explanation of this finding is in another study that applied the Apgar score during surgery, intraoperative hypotension (mean systolic arterial pressure, 40mm $\mathrm{Hg}$ ) also predicted cardiovascular events and other postoperative complications as well. [44]

Decreasing MACEs in TEA group and decreased fentanyl consumption were reflected on ICU and hospital stay as proved by Bouman et al. who reported that the patients in the epidural PCA group were discharged earlier in one and half days on average than the PCIA group. Also, patients in the epidural PCA group started ambulation earlier than in the PCIA group [45].

\section{CONCLUSION}

Perioperative PCEA in cardiac risky patients subjected to major gastrointestinal cancer surgery reduced significantly postoperative major adverse cardiac events with better pain control in comparison with perioperative PCIA analgesia.

\section{REFERENCES}

[1] Devereaux PJ, Chan MTV, Alonso-Coello P, et al.: Association between postoperative troponin levels and 30-day mortality among patients undergoing non cardiac surgery. JAMA 2012; 307:2295-304.

[2] Kheterpal S, O'Reilly M, Englesbe J, et al. Preoperative and intraoperative predictors of cardiac adverse events after general, vascular, and urological surgery. Anesthesiology. 2009;110(1):58-66.

[3] Lansky AJ, Stone GW. Periprocedural myocardial infarction: prevalence, prognosis, and prevention. Circ Cardiovasc Inters. 2010; 3: 602- 610.

[4] Lentine KL, Costa SP, Weir MR, et al. Cardiac disease evaluation and management among kidney and liver transplantation candidates: a scientific statement from the American Heart Association and the American College of Cardiology Foundation. Circulation. 2012; 126:617-63.

[5] Poldermans D, Bax JJ, Boersma E, et al. Guidelines for preoperative cardiac risk assessment and perioperative cardiac management in noncardiac surgery: the Task Force for Preoperative Cardiac Risk Assessment and Perioperative Cardiac Management in Non-cardiac Surgery of the European Society of Cardiology (ESC) and endorsed by the European Society of Anaesthesiology (ESA). Eur Heart J 2009; 30: 2769- 812 .

[6] Launay-Vacher V, Rey JB, Isnard-Bagnis C, Deray G, et al. Prevention of cisplatin nephrotoxicity: state of the art and recommendations from the European Society of Clinical Pharmacy Special Interest Group on Cancer Care. Cancer Chemother Pharmacol. 2008; 61(6): 903-909. 
Impact of Thoracic Epidural Analgesia on Major Adverse Cardiac Events (Maces) Following Major Gastrointestinal Cancer Surgeries

[7] Lee TH, Marcantonio ER, Mangione CM, et al. Derivation and prospective validation of a simple index for prediction of cardiac risk of major noncardiac surgery. Circulation 1999;100:10431049.

[8] Ford MK, Beattie WS, Wijeysundera DN. Systematic review: prediction of perioperative cardiac complications and mortality by the revised cardiac risk index. Ann Intern Med 2010; 152: 26-35.

[9] Oscarsson A, Fredrikson M, Sorliden M, et al. Predictors of cardiac events in high-risk patients undergoing emergency surgery.Acta Anaesthesiol Scand 2009, 53: 986- 994.

[10] Freise H, Van Aken HK. Risks and benefits of thoracic epidural anaesthesia. $\mathrm{Br} \mathrm{J}$ Anaesth 2011;107: 859-68.

[11] Imani F. Postoperative pain management. Anesth Pain 2011;1(1):6-7.

[12] Mangano DT, Hollenberg M, Fegert G, et al. Perioperative myocardial ischemia in patients undergoing noncardiac surgery--I: Incidence and severity during the 4 day perioperative period. The Study of Perioperative Ischemia (SPI) Research Group. J Am Coll Cardiol 1991, 17(4):843-850.

[13] Lentine KL, Costa SP, Weir MR, et al. Cardiac disease evaluation and management among kidney and liver transplantation candidates: a scientific statement from the American Heart Association and the American College of Cardiology Foundation. Circulation. 2012; 126:617-63.

[14] Poldermans D, Hoeks SE, Feringa HH. Preoperative risk assessment and Risk reduction before surgery. JA mColl Cardiol 2008;51:1913-1924.

[15] Skinner DL, Goga S, Rodseth RN, et al. A metaanalysis of intraoperative factors associated with postoperative cardiac complications.South Afr J Anaesth Analg 2012, 18:186-191.

[16] Mangano DT, Hollenberg M, Fegert G, et al. Perioperative myocardial ischemia in patients undergoing noncardiac surgery--I: Incidence and severity during the 4 day perioperative period. The Study of Perioperative Ischemia (SPI) Research Group. J Am Coll Cardiol 1991, 17(4):843-850.

[17] Priebe HJ. Triggers of perioperative myocardial ischaemia and infarction. Br J Anaesth 2004; 93:9-20.

[18] Waurick R, Van Aken H: Update in thoracic epidural anaesthesia. Best Pract Res Clin Anaesthesiol 2005; 19:201-13.

[19] Ogilvy AJ, Smith G - The gastrointestinal tract after anaesthesia. Eur J Anaesthesiol, 1995;12 (Suppl.10):35-42

[20] White PF, Kehlet H. Improving postoperative pain management: What are the unresolved issues? Anesthesiology 2010; 112: 220-5.

[21] Reichlin T, Hochholzer W, Stelzig C, et al. Incremental value of copeptin for rapid rule out of acute myocardial infarction. J Am Coll Cardiol 2009, 54:60-68.

[22] Snowden CP, Prentis J, Jacques B, et al. Cardiorespiratory fitness predicts mortality and hospital length of stay after major elective surgery in older people. Ann Surg. 2013; 257:999-1004.

[23] Yeager MP, Glass DD, Neff RK, et al. Epidural anesthesia and analgesia in high-risk surgical patients. Anesthesiology 1987; 66: 729-36.

[24] Loick HM, Schmidt C, Van Aken H, et al. High thoracic epidural anesthesia, but not clonidine, attenuates the perioperative stress response via sympatholysis and reduces the release of troponin $\mathrm{T}$ in patients undergoing coronary artery bypass grafting. Anesth Analg 1999; 88:701-9.

[25] Barrington M. J., R. Kluger, R. Watson, D. A. et al."Epidural anesthesia for coronary artery bypass surgery compared with general anesthesia alone does not reduce biochemical markers of myocardial damage,"Anesthesia and Analgesia, 2005 vol.100, no.4,pp.921 928.

[26] Mohamad Farouk Mohamad1 Montaser A Mohammad1 Diab F Hetta Eman Hasan Ahmed2 Ahmed A Obiedallah Alaa Ali M Elzohry; Thoracic epidural analgesia reduces myocardial injury in 
Impact of Thoracic Epidural Analgesia on Major Adverse Cardiac Events (Maces) Following Major Gastrointestinal Cancer Surgeries

ischemic patients undergoing major abdominal cancer surgery; Journal of Pain Research 2017:10 887-895.

[27] Bignami E, Landoni G, Biondi-Zoccai GG,et al. Epidural analgesia improves outcome in cardiac surgery: a meta-analysis of randomized controlled trials.J Cardiothorac Vasc Anesth 2009;23: 594- 9.

[28] Rodseth RN, Lurati Buse GA, Bolliger D, et al. The predictive ability ofpre-operative B-type natriuretic peptide in vascular patients for major adverse cardiac events: an individual patient data meta-analysis.JamColl Cardiol 2011,58:522-529.

[29] Liu SS, Wu CL. Effect of postoperative analgesia on major postoperative complications: a systematic update of the evidence. Anesth Analg 2007; 104:689-702.

[30] Popping DM, Zahn PK, Van Aken HK, et al. Effectiveness and safety of postoperative pain management: a survey of 18925 consecutive patients between 1998 and 2006 (2nd revision): a database analysis of prospectively raised data. Br J Anaesth 2008;101: 832-40.

[31] Blomberg S, Emanuelsson H, Kvist $\mathrm{H}$ et al.: Effects of thoracic epidural anesthesia on coronary arteries and arterioles in patients with coronary artery disease. Anesthesiology 1990; 73: 840-847.

[32] Schmidt C, Hinder F, Van Aken H,et al. The effect of high thoracic epidural anesthesia on systolic and diastolic left ventricular function in patients with coronary artery disease. Anesth Analg 2005;100: 1561-9.

[33] Svircevic V, Nierich AP, Moons KG, et al. Thoracic epidural anesthesia for cardiac surgery: a randomized trial.Anesthesiology 2011;114: 262-70.

[34] Mohamed SA, Fares KM, Hasan-Ali H, et al. The Effect of Anesthetic Technique on Cardiac Troponin-T and Systemic Inflammatory Response after Major Abdominal Cancer Surgery. J Anesthe Clinic Res 2013; 4: 296. doi:10.4172/21556148.1000296.

[35] Kessler P., T. Aybek, G. Neidhart et al. “Comparison of three anesthetic techniques for off-pump coronary artery bypass grafting: general anesthesia, combined general and high thoracic epidural anesthesia, or high thoracic epidural anesthesia alone,"Journal of Cardiothoracic and Vascular Anesthesia, 2005 ; vol.19,no.1,pp.32-39.

[36] Berendes E, C. Schmidt, H. van Aken et al., "Reversible cardiac sympathectomy by high thoracic epidural anesthesia improves regional left ventricular function in patients undergoing coronary artery bypass grafting: a randomized trial, "Archives of Surgery, 2003;vol.138, no. 12,pp. 1283-1290.

[37] Fillinger M. P., M. P. Yeager, T. M. Dodds, M. F. et al. "Epidural anesthesia and analgesia: effects on recovery from cardiac surgery,"Journal of Cardiothoracic and Vascular Anesthesia, 2002 vol.16, no.1,pp.15-20.

[38] Scott DA and McDonald WM. Assessment, Measurement and History. In Textbook of Clinical Pain Management 2nd edition. Macintyre PE, Rowbotham D, Walker S. Acute Pain; 2008.

[39] Moltner A, Holzl R, Strian F - Heart rate changes as an autonomic component of the pain response. Pain, 1990; 43(1):81-89.

[40] Giroban L, Dolinski SY, Zvara DA et al. -Thoracid epidural analgesia: its role in posthoracotomy atrial arrhythmias. J Cardoth Vasc Anest, 2000; 14: 662-665.

[41] Amar D, Zhang H, Heerdt PM, et al. Statin use is associated with a reduction in atrial fibrillation after noncardiac thoracic surgery independent of C-reactive protein. Chest 2005; 128: 3421-7.

[42] Amar D, Zhang H, Miodownik S, et al. Competing autonomic mechanisms precede the onset of postoperative atrial fibrillation.J Am Coll Cardiol 2003; 42: $1262-8$.

[43] Elzohry AAM, Abd-El-moniem Bakr M, Mostafa GM, Mohamad MF, Ahmed EH (2018) Continuous Perioperative Thoracic Epidural FentanylBupivacaine Infusion vs. Continuous Perioperative Fentanyl Intravenous Infusion in Patients Undergoing Major Upper Abdominal Cancer Surgeries. J Pain Manage Med 4: 132. 
Impact of Thoracic Epidural Analgesia on Major Adverse Cardiac Events (Maces) Following Major Gastrointestinal Cancer Surgeries

[44] Gawande AA, Kwaan MR, Regenbogen SE, et al. [45] Bouman EA, Theunissen M, Bons SA, et al. An Apgar score for surgery.J Am Coll Surg 2007; 204: 201-8.

Reduced incidence of chronic postsurgical pain after epidural analgesia for abdominal surgery. Pain Practice 2013 doi: 10.1111/papr.12091

Citation: Alaa Ali M. Elzohry, Mohamad Farouk. Mohamad et al. Impact of Thoracic Epidural Analgesia on Major Adverse Cardiac Events (Maces) Following Major Gastrointestinal Cancer Surgeries. Archives of Anesthesiology. 2019; 2(1): 01-14

Copyright: (C) 2019 Alaa Ali M. Elzohry, Mohamad Farouk. Mohamad et al. This is an open access article distributed under the Creative Commons Attribution License, which permits unrestricted use, distribution, and reproduction in any medium, provided the original work is properly cited. 